\title{
FATIGUE FAILURE OF OSTEOCYTE CELLULAR PROCESSES: IMPLICATIONS FOR THE REPAIR OF BONE
}

\author{
Clodagh Dooley ${ }^{1,2, *}$, Daniel Cafferky ${ }^{1}$, T. Clive Lee ${ }^{1,3}$ and David Taylor ${ }^{1}$ \\ ${ }^{1}$ Trinity Centre for Bioengineering, Trinity College, Dublin, Ireland \\ ${ }^{2}$ Centre for Microscopy and Analysis, Trinity College, Dublin, Ireland \\ ${ }^{3}$ The Royal College of Surgeons in Ireland, Dublin, Ireland
}

\begin{abstract}
The physical effects of fatigue failure caused by cyclic strain are important and for most materials well understood. However, nothing is known about this mode of failure in living cells. We developed a novel method that allowed us to apply controlled levels of cyclic displacement to networks of osteocytes in bone. We showed that under cyclic loading, fatigue failure takes place in the dendritic processes of osteocytes at cyclic strain levels as low as one tenth of the strain needed for instantaneous rupture. The number of cycles to failure was inversely correlated with the strain level. Further experiments demonstrated that these failures were not artefacts of our methods of sample preparation and testing, and that fatigue failure of cell processes also occurs in vivo. This work is significant as it is the first time it has been possible to conduct fatigue testing on cellular material of any kind. Many types of cells experience repetitive loading which may cause failure or damage requiring repair. It is clinically important to determine how cyclic strain affects cells and how they respond in order to gain a deeper understanding of the physiological processes stimulated in this manner. The more we understand about the natural repair process in bone the more targeted the intervention methods may become if disruption of the repair process occurred. Our results will help to understand how the osteocyte cell network is disrupted in the vicinity of matrix damage, a crucial step in bone remodelling.
\end{abstract}

Keywords: Osteocytes; Microcracks; Cell fatigue failure; Osteocyte fatigue failure; Scissors model; Bone remodelling.

\footnotetext{
*Address for correspondence:

Clodagh Dooley

Centre for Microscopy and Analysis

Trinity College Dublin

Dublin 2, Ireland
}

E-mail: dooleycl@tcd.ie

\section{Introduction}

The mechanical behaviour of living cells, i.e., the deformation and failure of the cell membrane and cytoskeleton as a result of externally applied forces, is a topic of considerable interest in the field of mechanobiology. In recent years, many studies have been conducted to measure the elastic and viscoelastic deformations of cells and cell membranes, using advanced techniques such as atomic force microscopy (Kuznetsova et al., 2007). However, very few researchers have studied damage and failure, largely owing to the practical difficulties of this type of experimentation. Exceptionally, Nichol and Hutter measured the failure strain of the lipid bilayer (Nichol and Hutter, 1996) and Sheetz et al. described blebbing: a cell damage phenomenon that results in the complete rupture of the cell membrane (Sheetz et al., 2006 ).

Many cell types experience cyclic strains of significant magnitude as they function and travel through the body. For example, fluid shear forces acting on endothelial cell membranes are estimated to cause strains of $22 \%$ (Mofrad and Kamm, 2010). Cyclic strain is a necessary signal for cell differentiation: for example, cells of the vascular and musculature systems will only begin to differentiate if they regularly experience more than $5 \%$ strain (Mofrad and Kamm, 2010). Our motivation for the work described arose from the need to better understand the mechanical environment of osteocytes and their role in the detection and repair of microcracks in bone. Since the work of Frost (Frost, 1960) it has been known that bone contains microcracks, which he suggested were the result of cyclic loading of bone as would occur with repetitive movements such as walking. These cracks are typically elliptical in shape, with dimensions of $100 \mu \mathrm{m} \mathrm{x}$ $400 \mu \mathrm{m}$, oriented close to the longitudinal axis of the bone (O’Brien et al., 2000). Without repair, these cracks will grow to cause stress fractures within a matter of months (Taylor, 2011). Repair is carried out by basic multicellular units (BMUs) (Eriksen and Langdahl, 1995; Jilka, 2003) which are known to target areas of microdamage (Burr and Martin, 1993; Burr, 2002; Lee et al., 2002), implying that the osteocyte network is able to detect the presence of microcracks and signal to the cells of the BMU to initiate the repair process. There has been considerable debate in the literature as to how osteocytes detect microcracks (Frost, 1987; Cowin et al., 1991; Dunstan et al., 1993; Mori et al., 1997; Cowin, 2002; Bonewald, 2011). A number of hypotheses have been put forward but to date no complete mechanism has been demonstrated. Possible mechanisms include the detection of changes in 
cell membrane strain, enhanced by local fluid flow (You et al., 2000; You et al., 2001) and the stimulation of the osteocyte's primary cilium (Malone et al., 2007; Anderson et al., 2008; Temiyasathit and Jacobs, 2010). Apoptosis has been shown to increase in the vicinity of microdamage, though the mechanism which causes this is unclear (Noble et al., 1997; Schaffler and Jepsen, 2000; Verborgt et al., 2000; Noble, 2003).

Another proposed mechanism for damage-stimulated repair, developed by our research group, is the so-called "scissors model". We proposed that the dendritic cellular processes which link osteocytes could be ruptured at the point where they passed across a microcrack face as a result of applied cyclic strain, giving rise to a signal which might stimulate and direct preosteoclasts and BMUs (Taylor et al., 2003; Hazenberg et al., 2006), either directly or possibly via apoptosis of cells local to the damaged region (Kennedy et al., 2012). We found examples of osteocyte cellular processes which had ruptured at crack faces, and showed that the proportion of ruptured cell processes was greater in longer cracks and in cracks subjected to higher stresses (Dooley et al., 2012). We showed that cell process rupture could occur in vivo but that crack propagation in the absence of cyclic strain did not cause damage to the cellular material, a finding that was supported by the observations of Colopy et al. who noted disruptions to the osteocyte network in the vicinity of microdamage, after cyclic loading (Colopy et al., 2004).

In our original model we proposed that the rupture of these processes would occur instantaneously, by a scissorlike cutting motion as the crack faces were simultaneously pressed together and sheared over each other. The present investigation arose because we wanted to investigate this failure mechanism in more detail, and in particular to consider whether rupture did occur instantaneously. Fatigue failure, whereby failure occurs at relatively low stresses if the stress is repeatedly applied and removed in successive cycles, occurs in many materials. It is also a highly plausible mechanism for the rupture of osteocyte cell processes given the cyclic nature of movement, and therefore warranted investigation.

The aims of the present study were:

1. To directly observe the deformation and failure of individual osteocyte cellular processes spanning microcracks, including both instantaneous failure under monotonic loading and fatigue failure under cyclic loading.

2. To investigate the effect of the magnitude of cyclic loading and the number of cycles on the rupture of osteocyte cell processes.

\section{Materials and Methods}

Bovine cortical bone was obtained from skeletally mature, disease free animals, exposed to normal in vivo loading and slaughtered within $24 \mathrm{~h}$ of sample processing (Kepac group, Clonee, Ireland). Two sample types were prepared immediately after acquiring refrigerated bone. A different animal source was used for each of the testing methods. The first set were stained using a technique developed by

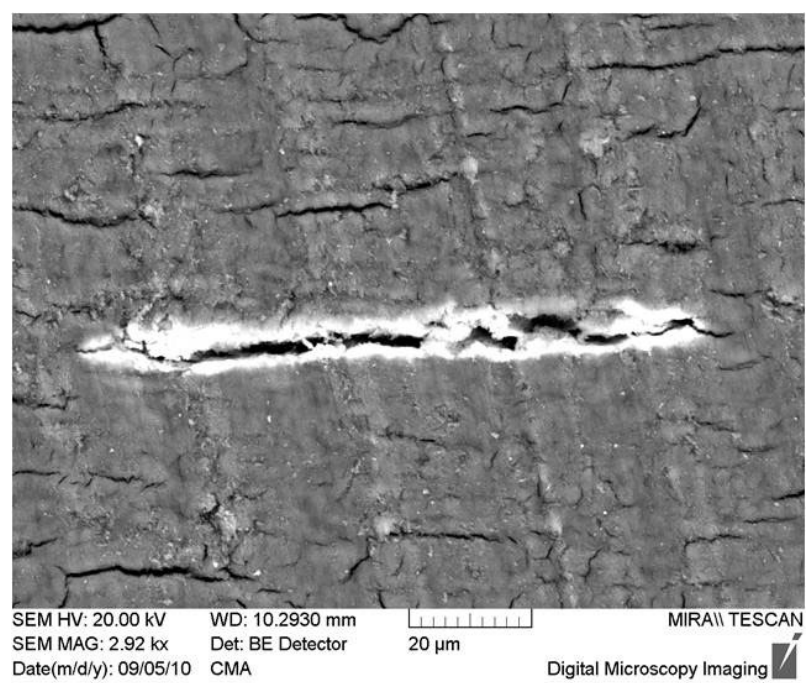

Fig.1. Scanning electron microscope image using a backscatter electron detector showing a halo of heavy metal stain surrounding a microcrack. This indicates that the microcrack formed in vivo and is not an artefact of sample processing.

Schaffler et al. (Schaffler et al., 1994), in order to confirm that the microcracks being examined had occurred in vivo rather than during sample preparation or testing. A heavy metal stain was applied by placing the samples into a solution of $70 \%$ acetone containing a lead-uranyl complex (20\% lead acetate; $8 \%$ uranyl acetate). They were then rinsed in $70 \%$ acetone and placed into a solution of $1 \%$ ammonium sulphide in acetone for 7 days to form lead and uranyl sulphide precipitates. This resulted in the original in vivo microcracks becoming stained in a manner which was visible in the scanning electron microscope when using a backscatter electron detector. This allowed us to distinguish in vivo microcracks from artefact microcracks which formed during preparation, in particular during sample drying (Fig. 1).

Once stained the samples were subjected to further loading in vitro. Six samples, cut from mid-way along the bone diaphysis, were machined into cylindrical, waisted, dog bone shaped specimens. The cylindrical samples were created using a high-speed-steel core-milling cutter. Every effort was made to reduce the level of damage caused by machining. Firm gripping without damage was achieved by embedding the samples in plaster held within aluminium box-section moulds. Continuous liquid cooling was used throughout to prevent overheating of the material. Visual inspection of the final specimens showed good surface finish with no obvious machining defects. A previous paper by Taylor et al. examined the effect of machining dog bone shaped specimens on sample damage and identified only small amounts of surface damage and this damage did not appear to initiate fatigue damage (Taylor et al., 1999). The dog bone shaped samples were loaded in cyclic compression using a protocol which has been described previously (O'Brien et al., 2002). Bone hydration was maintained using a bath containing phosphate buffered saline (PBS). One control sample was not exposed to cycling and was used to represent in vivo 
loading conditions. The stress cycle chosen was sinusoidal compression with a ratio between the maximum stress and minimum stress of 10 to 1 . Samples were subjected to a stress range of $80 \mathrm{MPa}$ at increasing cycle numbers (1, $100,1000,10,000$ or 100,000 cycles). To put these values in context, the maximum stress normally experienced in vivo is of the order of $40 \mathrm{MPa}$ and the number of cycles experienced in vivo is of the order of one million per year. Bone samples loaded to $80 \mathrm{MPa}$ typically fail after about 100,000 cycles in vitro (O’Brien et al., 2002).

After testing, samples were cut from the waisted middle of the dog bone into transverse slices (approximately 8 sections per sample), $100 \mu \mathrm{m}$ thick, using a diamond saw (Struers, Minitron) and ground flat using silicon carbide paper. Deionised water was used as a lubricant while cutting and grinding. After, sections were fixed in $4 \%$ paraformaldehyde solution for $24 \mathrm{~h}$ at $4{ }^{\circ} \mathrm{C}$ and washed three times in PBS. Following this, they were alcohol dehydrated, critical point dried, mounted using carbon cement and carbon coated for examination in a scanning electron microscope (SEM) (Tescan MIRA XMU; Tescan, Brno, Czech Republic) using a backscattered electron detector.

Fifty microcracks which had formed in vivo, identified by their heavy metal stain, were examined from each of the six groups (the control (in vivo) sample plus five samples loaded with different numbers of cycles). Five

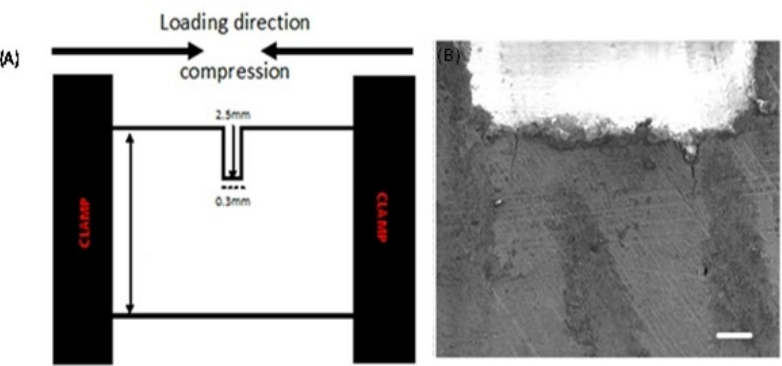

Fig 2. (a) Diagram showing the orientation of the bone sample in the in situ loading stage. (b) SEM image of typical microcracks propagated from a notch.

to ten microcracks were examined from each of the approximate eight sections, avoiding the inner or outer edge of the section and therefore avoiding areas where the machining of samples could have caused damage, until a total of fifty microcracks were counted for each strain level. These microcracks were all formed in vivo due to normal physiological loading as a result of everyday movement. The long axis of the sample was cut from the long axis of the bone so microcrack orientation, and therefore the compressive strain acting on the microcrack and the surrounding osteocytes, would be representative of that experienced by a limb and it's naturally formed

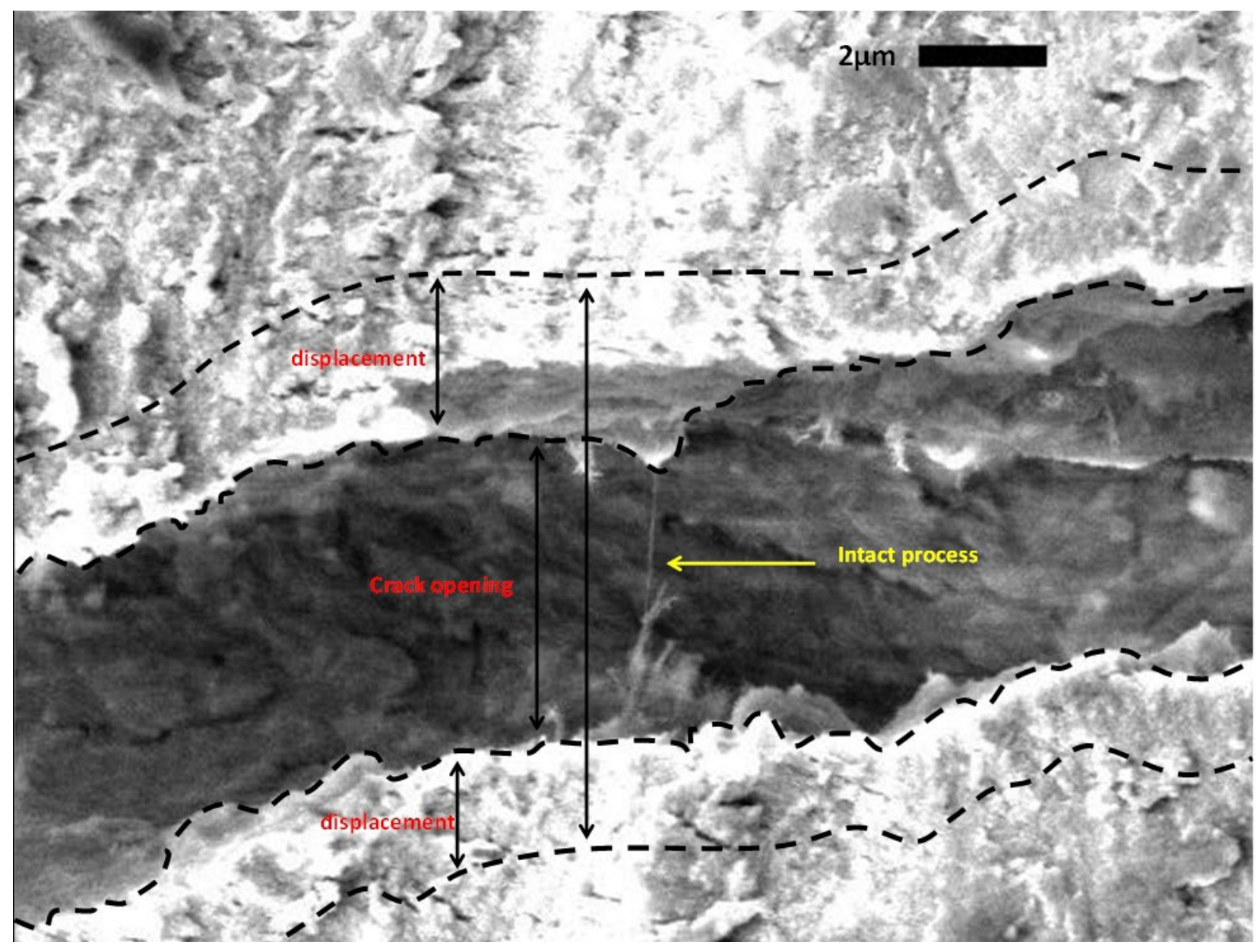

Fig. 3. SEM image of part of a microcrack, showing an intact process, indicating the crack opening displacement under compressive loading and (schematically) how this changed when the loading was removed. 
microcracks throughout cyclic compressive loading due to movement.

Microcracks also formed as a result of the sample preparation process: fifty of these unstained, artefact microcracks were also examined in the control (in vivo) sample. Again, five to ten microcracks were examined from each of the approximately 8 sections, avoiding the inner or outer edge, until a total of fifty microcracks were counted. The use of the in vivo sample ensured that the artefact microcracks had not been exposed to any cyclic strain. SEM images were taken at a low magnification to allow accurate measuring of the crack length and width, high magnification images were also captured to allow counting of the intact cellular processes spanning the crack. A selection of the intact process counts were verified by a second, independent observer to ensure accuracy.

More detailed examinations were achieved using an insitu straining stage (Deben 200N Straining Stage; Deben, East Grinstead, UK) which allowed us to apply forces to samples inside the SEM and observe the resulting effect on the microcrack and the surrounding osteocytes. As the microcracks being observed were created during testing, there was no need for bulk heavy metal staining. The samples were fixed in $4 \%$ paraformaldehyde solution for $24 \mathrm{~h}$ at $4{ }^{\circ} \mathrm{C}$ and washed three times in phosphate buffered saline (PBS). They were alcohol dehydrated and critical point dried. Rectangular shaped samples, approximately $20 \mathrm{~mm} \times 10 \mathrm{~mm} \times 1 \mathrm{~mm}$ in size, were cut from the longitudinal axis of the bone; all samples were notched in the centre of the long axis of the sample, using a diamond saw. Fig. 2 indicates notch length, direction and loading direction in relation to the bone axis. The samples were clamped between the two arms of the straining stage and compressive, cyclic loading was applied.

Two types of testing were performed on these sample types. In the first a crack was propagated from the notch by gently tapping the clamped bone sample. The stage, with the sample in place, was then placed into the SEM chamber and subjected to 100 cycles of compressive stress, cycling between a maximum of $18 \mathrm{MPa}$ and a minimum of $0.1 \mathrm{MPa}$. Images of all the individual intact processes present along the crack were taken prior to any compression, after one cycle and after 100 cycles.

In the second set of tests, the crack was generated and loaded cyclically as before. However in this case the crack opening displacement in the vicinity of each process was measured at the maximum and minimum load of every cycle, up to the point where the process ruptured. Fig. 3 indicates how the crack opening displacement was defined for the crack, and schematically how it changed during the loading cycle.

All statistical analysis was performed using SPSS (Chicago, IL, USA). ANOVA was used to determine if there was a statistical difference between data sets. Normal distribution was determined using the Shapiro-Wilk test and equal variance was determined using the Levene test for homogeneity of variance prior to statistical analysis.

\section{Results}

SEM imaging clearly showed fibres spanning each microcrack (Fig. 4). These fibres, which have a distinctive morphology making them easy to identify in the SEM, had been observed previously and were identified as osteocyte's cellular processes using phalloidin, an antibody stain specific to f-actin (Hazenberg et al., 2006). Fig. 5 shows the average number of intact osteocyte processes per millimetre of crack length, for 50 cracks, in each testing condition. There were statistically significant differences $(p<0.05)$ between the results for the artefact cracks, the in vivo control samples and those samples cycled at $80 \mathrm{MPa}$ for 100 cycles or more. There was also a significant difference in the number of intact osteocyte cell processes between the samples exposed to one cycle at $80 \mathrm{MPa}$ and those exposed to larger numbers of cycles. No statistically significant difference was found between the in vivo sample and the single cycled sample, or amongst the four groups tested at 100,1000,10,000 and 100,000 cycles. The strained samples were separated into long $(>100 \mu \mathrm{m})$ and short cracks $(\leq 100 \mu \mathrm{m})$ and were compared using ANOVA. It was found that shorter cracks had statistically
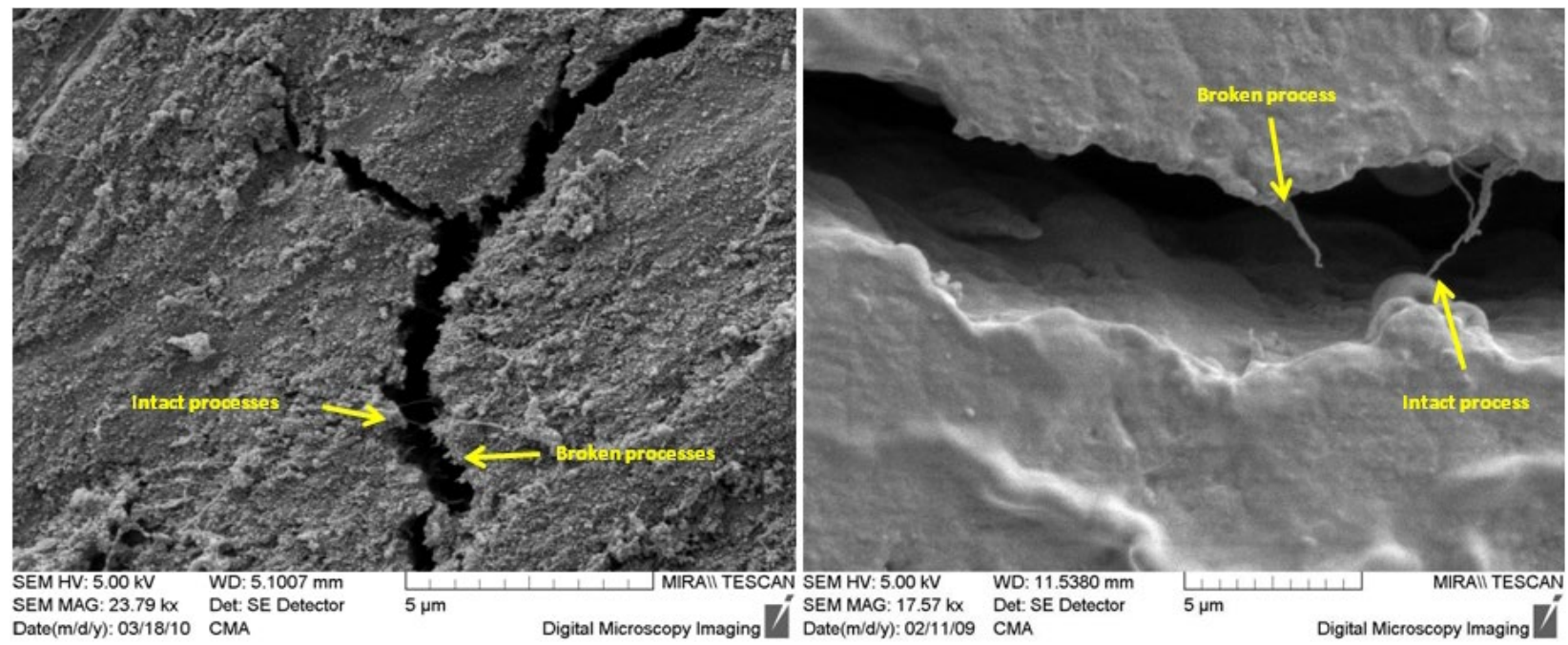

Fig.4. SEM images showing examples of intact and broken osteocyte processes spanning microcracks. 


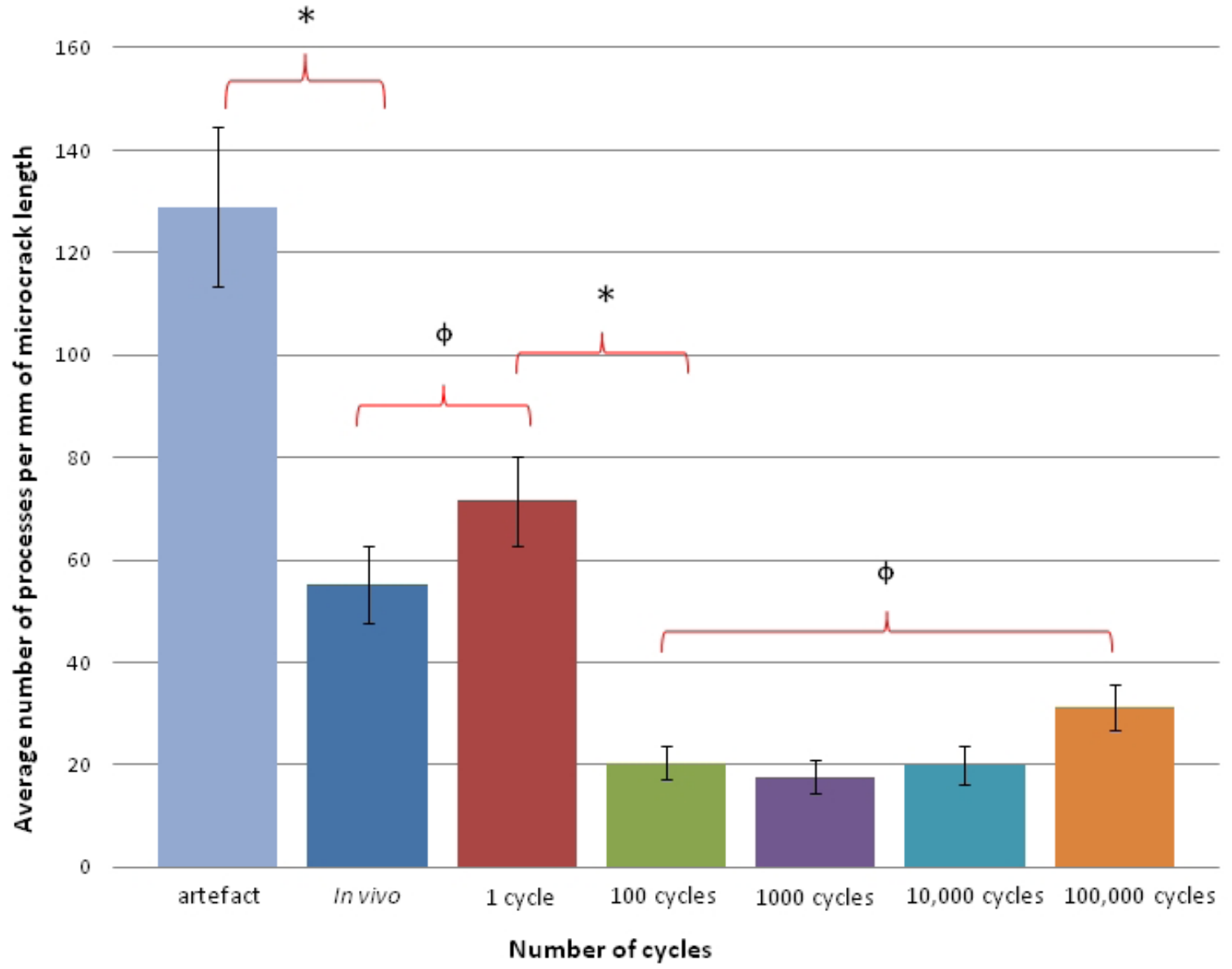

Fig 5. Results for normal, fully hydrated bone samples, showing the number of intact processes per millimetre of crack length. Artefact cracks are those generated during sample preparation which have never experienced cyclic loading. In vivo microcracks have experienced normal physiological stresses. The other samples were cycled at a higher stress range $(80 \mathrm{MPa})$ for $1,100,1000,10,000$ or 100,000 cycles. $*$ denotes a statistically significant difference between data sets and $\phi$ denotes no statistically significant difference between data sets. Statistical significance is defined as a $p$ value of $\leq 0.05$ using ANOVA.

Table 1. This table indicates the statistical differences ( $p$ values) in the number of intact cell processes in hydrated samples with an increasing number of cycles of compressive loading (see Fig. 5).

\begin{tabular}{|l|c|c|c|c|c|c|}
\hline & artefact & $\mathbf{1}$ cycle & $\begin{array}{c}\mathbf{1 0 0} \\
\text { cycles }\end{array}$ & $\begin{array}{c}\mathbf{1 0 0 0} \\
\text { cycles }\end{array}$ & $\begin{array}{c}\mathbf{1 0 0 0 0} \\
\text { cycles }\end{array}$ & $\begin{array}{c}\mathbf{1 0 0 0 0 0} \\
\text { cycles }\end{array}$ \\
\hline control & 0.165 & 0.452 & $<0.05$ & $<0.05$ & $<0.05$ & $<0.05$ \\
\hline artefact & $\mathrm{n} / \mathrm{a}$ & $<0.05$ & $<0.05$ & $<0.05$ & $<0.05$ & $<0.05$ \\
\hline $\mathbf{1}$ cycle & $<0.05$ & $\mathrm{n} / \mathrm{a}$ & $<0.05$ & $<0.05$ & $<0.05$ & 0.1 \\
\hline $\mathbf{1 0 0}$ cycles & $<0.05$ & $<0.05$ & $\mathrm{n} / \mathrm{a}$ & 0.902 & 0.567 & 0.747 \\
\hline $\mathbf{1 0 0 0}$ cycles & $<0.05$ & $<0.05$ & 0.902 & $\mathrm{n} / \mathrm{a}$ & 0.997 & 0.111 \\
\hline $\mathbf{1 0 0 0 0}$ cycles & $<0.05$ & $<0.05$ & 0.567 & 0.997 & $\mathrm{n} / \mathrm{a}$ & 0.021 \\
\hline $\mathbf{1 0 0 0 0 0}$ cycles & $<0.05$ & 0.1 & 0.747 & 0.111 & 0.021 & $\mathrm{n} / \mathrm{a}$ \\
\hline
\end{tabular}

more intact processes remaining than long cracks in all samples $(p \leq 0.05)$.

Microcrack opening width was measured at the widest central point of each microcrack to determine whether crack opening alone could have caused the rupture of the osteocyte cell processes (Fig. 6). There was a statistical difference between the width of artefact microcracks and the in vivo strained sample, 1 cycle, 100 cycles and 1000 cycled samples. No statistical difference was found between the in vivo, 1 cycle, 100 cycle and 1000 cycle samples. A reduction in the microcrack openings was noted at between 100 cycle sample and the 1000 cycle sample but the reduction was not statistically significant. The reduction in microcrack opening between 100 cycles and 10,000 cycles was statistically significant. These results indicate that cyclic loading does not cause a progressive increase in crack width, implying that the failure of processes is a true fatigue effect and not due to static stretching.

Eleven microcracks were tested for 100 loading cycles in situ in the SEM. Fig. 7 shows the number of intact processes over the course of the experiment. Of a total of 74 intact processes at the start of the test, 17 processes 


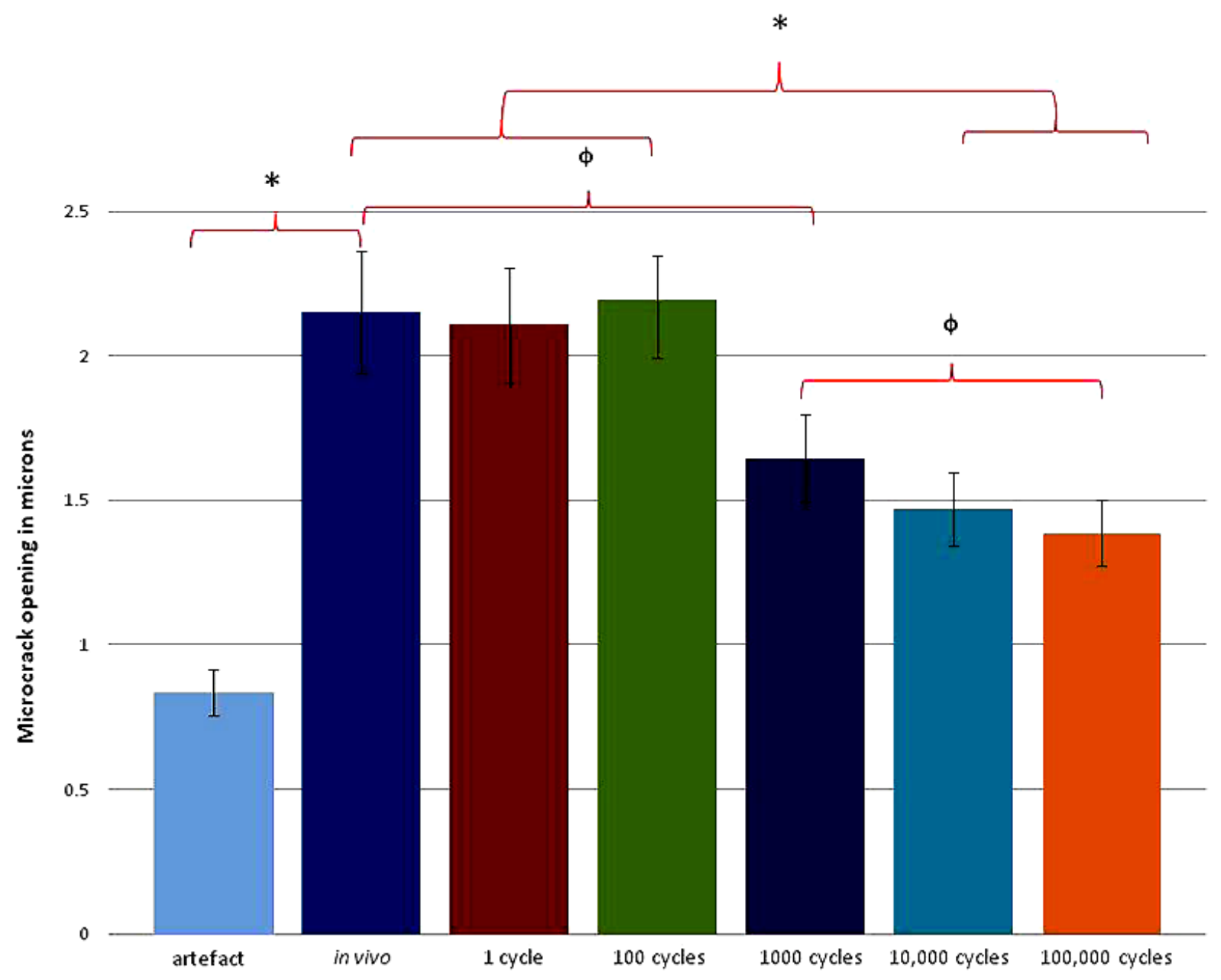

Number of cycles

Fig. 6. Results for normal, fully hydrated bone samples, showing the average microcrack opening in microns. Artefact cracks are those generated during sample preparation which have never experienced cyclic loading. In vivo microcracks have experienced normal physiological stresses. The other samples were cycled at a higher stress range (80 MPa) for $1,100,1000,10,000$ or 100,000 cycles. $*$ denotes a statistically significant difference between data sets and $\phi$ denotes no statistically significant difference between data sets. Statistical significance is defined as a $p$ value of $\leq 0.05$ using ANOVA.

failed instantaneously during the first loading cycle, whilst a further 40 failed by fatigue after 100 cycles. A further eight samples were cycled in the SEM whilst observing individual processes in detail. Fig. 8 shows an example of the variation in crack opening displacement cycle-by-cycle in the vicinity of an osteocyte process, which in this case ruptured after 4 cycles. Compressive loading tended to close the cracks, but not completely. Over time the average opening tended to decrease but the cyclic displacement range (i.e., the difference between the maximum and minimum in successive cycles) remained approximately constant. Fig. 9 shows the number of cycles to cell process failure as a function of the average displacement range, measured at the crack faces, in the vicinity of individual processes. Monotonic rupture on the first cycle occurred once, at a displacement of about $2 \mu \mathrm{m}$; reducing the displacement produced failure after increasing numbers of cycles, with failure of cell processes occurring even at displacements less than one tenth of that necessary for monotonic rupture. Changes in the microcrack length were recorded during in situ cyclic compressive testing to see if there was an increase in microcrack length. It was found that on average microcracks increased by only $9.7 \%$ as a result of cycling. This increase would not explain the large number of broken processes.

\section{Discussion}

This work has demonstrated that osteocyte processes will fail as a result of cyclic strains applied at a magnitude which is not sufficient to cause instantaneous rupture. We believe that this is the first evidence of fatigue failure of cellular material in any type of cell. It is conventional in fatigue studies to plot these data logarithmically, as shown here, and to apply a linear trendline, implying the following relationship between cyclic strain $(\Delta \varepsilon)$ and the number of cycles to failure $\left(N_{f}\right)$.

$$
N_{f}=\frac{C}{\Delta \varepsilon^{n}}(1)
$$

Here $C$ and $n$ are constants. The line shown (Fig. 9) has a good fit to the data $\left(R^{2}=0.77\right)$ with the exponent $n=0.75$. 


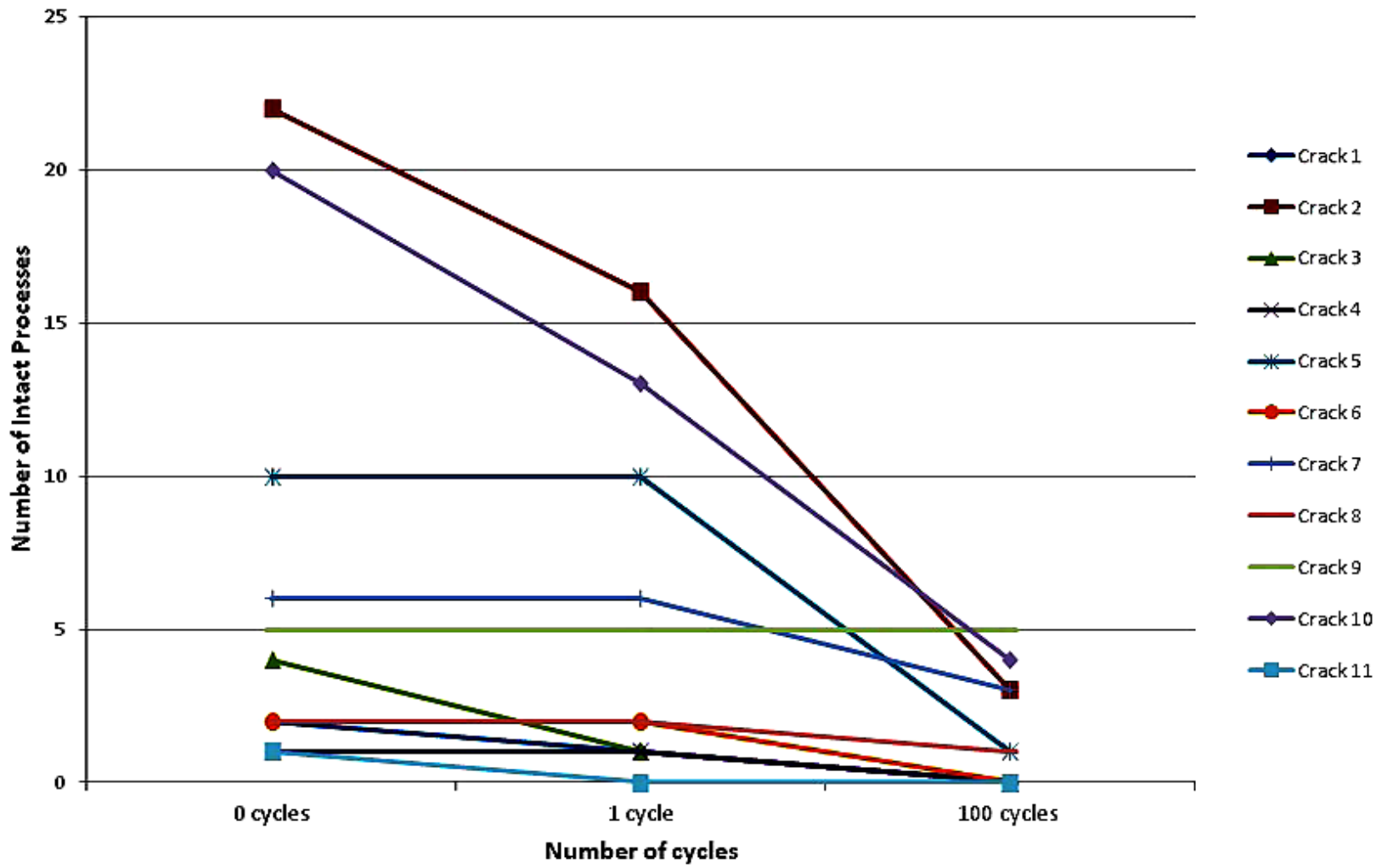

Fig. 7. Changes in the number of intact osteocyte processes for cracks subjected to cyclic loading in situ in the SEM.

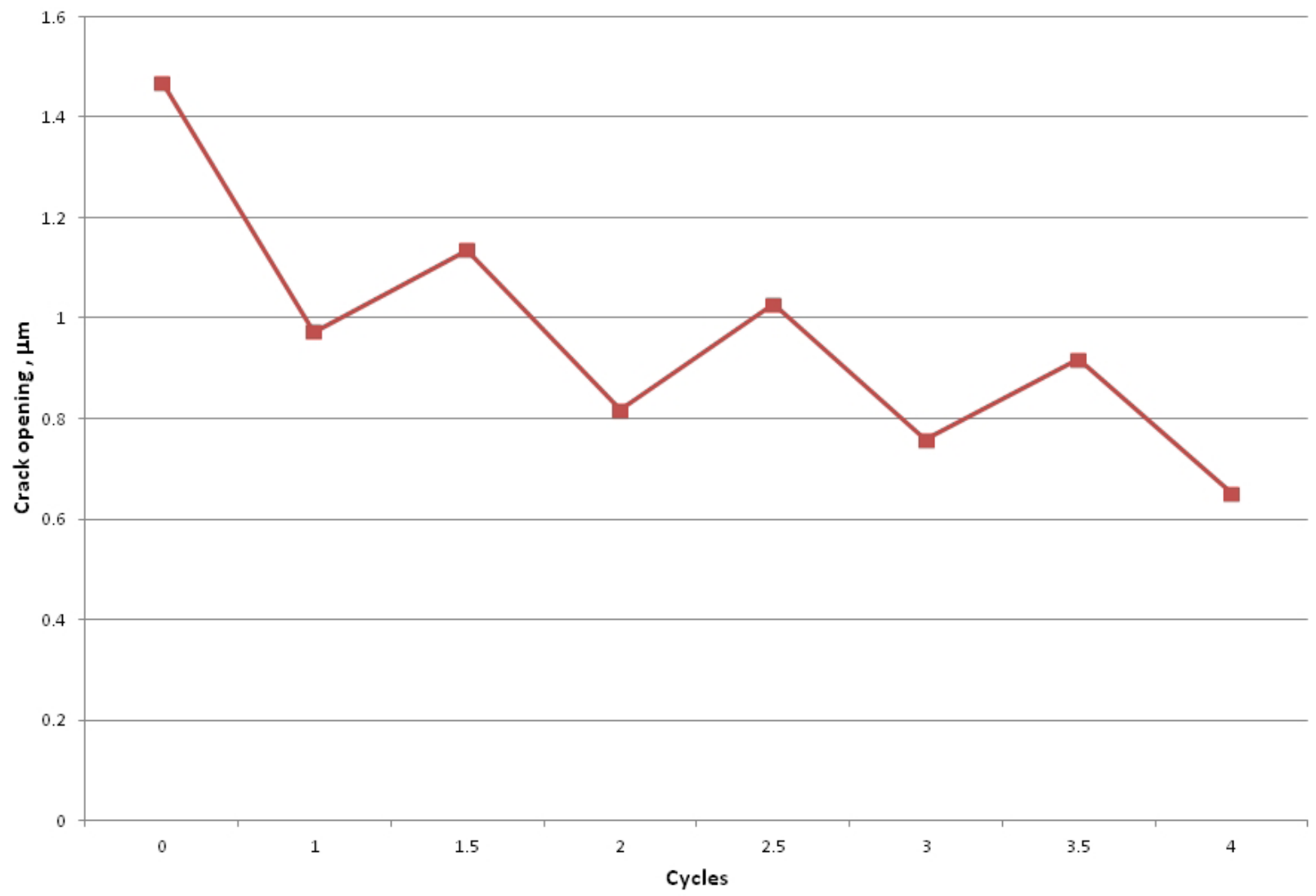

Fig. 8. An example of results from in situ cyclic loading, showing the variation of crack opening displacement cycleby-cycle in the vicinity of an osteocyte process which ruptured after four cycles. 


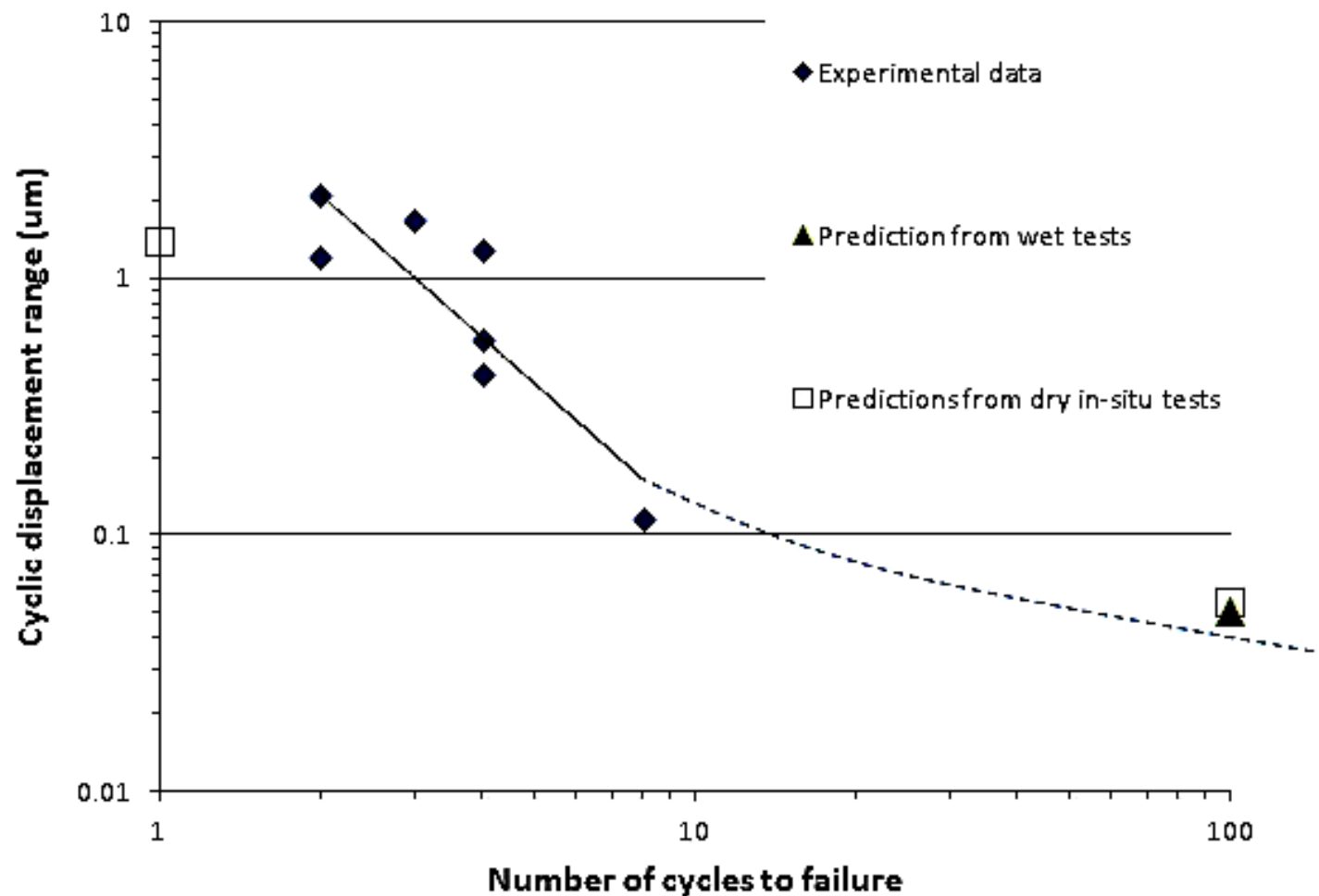

Fig. 9. Results from the in situ fatigue tests on individual osteocyte processes, showing the number of cycles to failure as a function of cyclic displacement range. Also included are three estimated results obtained by analysis of the 100 cycle data from dry and wet samples.

Compared to data on other materials this is a relatively low value of $n$, but not unprecedented (Boller and Seeger, 1987).

A limitation of this study was the small number of cycles to cell process failure: up to 8. Smaller cyclic displacements will likely cause failure after increased numbers of cycles, but our method was not able to reliably measure displacements less than $0.1 \mu \mathrm{m}$. However, by testing samples using the same in situ method but with an increase in cycle numbers to 100 we demonstrated the prevalence of fatigue failure of cell processes (Fig. 7). Of a total of 74 intact processes initially, 17 ruptured after one cycle of loading whilst 57 had ruptured after 100 cycles, showing that process rupture is very common and that fatigue is the dominant failure mechanism of osteocyte cell processes.

Another mode of long term failure which may occur in cell processes is creep. Creep is a gradual increase in strain as a result of an applied stress. This can cause failure after a period of time, but it is unlikely to be occurring in the present tests because (as Fig. 8 shows) we were subjecting processes to an applied strain (not stress), and this strain was gradually decreasing over time. Under these conditions creep will act to reduce the applied stress and thus protect the process from creep failure.

There was a concern that the methods of sample preparation and the dry, vacuum environment of the SEM chamber may have altered the properties of the material and thus affected the strength of the results. This was addressed by also testing samples in their normal, nonfixed, hydrated condition (Fig. 5) prior to sectioning, fixing and dehydrating. This ensured that all osteocyte process rupture observed occurred in cells that were in a condition as close to their natural, in vivo state as possible. Applying 100 cycles caused a reduction of $62 \%$ in the number of intact processes, whilst one cycle had no statisticallysignificant effect. The $62 \%$ failure rate in the hydrated sample is comparable to the $77 \%$ failure rate after the same number of cycles for the dry, fixed samples tested in the SEM, suggesting that fixation and drying has relatively little effect on the results.

Applying further cycles up to 100,000 had no significant effect. This is probably due to the fact that, after 100 cycles, most the processes which were going to fail have already failed. The strains experienced by individual process vary depending on their position with respect to the crack tips and due to random local variations. As the number of cycles increases the cracks tend to close up (see Fig. 6), reducing the average strain on process.

Fixation has been shown to affect the elasticity of cell membranes by decreasing it 10 fold (Kuznetsova et al., 2007). It is however the case that fatigue at low numbers of cycles is dependent on the applied strain range, rather than the stress range. So increasing the elastic modulus of the cell membrane may have little effect since the loading is determined by the displacement of the crack faces. The above comparisons also address the concern that the heavy metal staining may have altered the cell membrane properties in a way that makes it unrepresentative of in vivo behaviour. It was not necessary to stain the cracks propagated in situ and as the results for both this test and the hydrated sample testing were comparable it enabled us to dismiss this as a limitation to the investigation. 
Artefact microcracks, which had not experienced any cyclic loading, had many more intact cellular processes than microcracks exposed to cyclic strain, even the low strain experienced by in vivo microcracks. On average they had 128.9 intact processes per millimetre of crack length, which is close to the number that would be expected if all processes were intact, according to our previous estimates (Dooley et al., 2012). This demonstrates two important findings, firstly, that our preparation techniques, namely drying, fixing and polishing, do not cause cell processes to fail. Secondly, comparing these results to those for cracks subjected to only in vivo stress levels (Fig. 5) shows that osteocyte cellular processes fail in vivo due to cyclic loading derived from normal daily activities. These results show that over half of the intact osteocyte cellular processes will rupture when exposed to even the low cyclic strain of normal physiological loading conditions, and while cells are alive and in their native condition.

Using a theoretical model which we developed previously (Taylor et al., 2003), we can estimate the crack face displacements during cyclic loading and thus add further data points to the fatigue results. The data on Fig. 7 are consistent with cell process failure within 1 cycle at a displacement of $1.4 \mu \mathrm{m}$ and within 100 cycles at a displacement of $0.054 \mu \mathrm{m}$ for the tests conducted inside the SEM vacuum chamber. For the tests on the non-fixed, hydrated samples, Fig.5 is consistent with cell process failure in 100 cycles at a displacement of $0.05 \mu \mathrm{m}$. These three estimated data points conform to the experimental data from individual processes (Fig. 9), suggesting that the results from the various tests are consistent and thus providing further evidence that our preparation techniques do not affect the fatigue behaviour of the cell processes.

Another limitation of our experiment is that we can measure only the cyclic displacement range, not the strain range, which is calculated by dividing the displacement by the total length of the material sample. We can only see the process as it passes across the crack so we don't know its total length. Given that bovine bone typically contains 30,000 osteocytes $/ \mathrm{mm}^{3}$ (Mullender et al., 1996) we can estimate that the average distance between cells is $32 \mu \mathrm{m}$. Taking account of cell diameter, a typical process will be about $10 \mu \mathrm{m}$ long, so the displacements in our tests will lead to strains in the range 1-20\%, higher if the process is attached to the matrix, reducing its effective length. As noted above, strains of this magnitude are known to occur in many types of cells.

These results provide further support for our "scissors" model of damage detection, albeit in a modified form in which the primary cause of cell process rupture is cyclic fatigue failure rather than instantaneous cutting. The low value of $n$ in equation 1 , and the consequent steep slope of the line on Fig. 9, has an interesting effect. Testing bone samples at $80 \mathrm{MPa}$, which is approximately half the stress needed for instantaneous failure, we find that most of the cellular processes have failed after 100 cycles, whilst (as noted above) the bone matrix itself has an average life of 100,000 cycles. Osteocyte process failure could possibly provide a very neat early warning system for bone and its cells to detect fatigue damage long before it becomes catastrophic. Our previous work has shown that as osteocyte cell networks are ruptured, cell signalling pathways known to stimulate targeted repair in bone are initiated (Mulcahy et al., 2011). With this further information we will be able to modify our theoretical model to more accurately predict the number of ruptured processes and simulate the dynamics of the damage/repair cycle.

This work is a fundamental study shedding light on a mechanism of failure which has not previously been observed for cells. Further work is needed to describe this phenomenon in more detail and investigate its clinical consequences. Microcracks have been linked to both skeletal fragility and stress fractures. Osteoporosis resulting from skeletal fragility is a major cost burden on health services, along with causing discomfort and health risks for those affected. This study shows that osteocyte processes can rupture by fatigue failure due to cyclic loading. This gives us an insight into how a bone may identify the presence of a microcrack and provides findings that fit with our group's hypothesis that the rupture of osteocyte cell processes can cause the release biochemical signals necessary for the stimulation of bone repair. This is the first step in, hopefully, understanding the complete picture of microcrack identification, assessing the risk to the bone and finally the initiation of repair. Understanding this process may help in the development of in an earlier and more targeted medical response to excessive microcrack formation.

This study provides the first ever quantitative evidence that fatigue occurs in any part of a cell, that it occurs at cyclic strains much less than the monotonic failure strain, and that it is the primary cause of failure for the dendritic processes of osteocytes in the vicinity of microcracks in bone. We hope that this initial study will stimulate others to conduct fatigue and fracture tests on osteocytes and other cell types which experience mechanical forces in vivo.

\section{Acknowledgements}

This work was conducted with financial support from Science Foundation Ireland under grant award number 08-RFP-ENM991.

\section{References}

Anderson CT, Castillo AB, Brugmann SA, Helms JA, Jacobs CR, Stearns T (2008) Primary cilia: Cellular sensors for the skeleton. Anat Rec 291: 1074-1078.

Boller C, Seeger T (1987) Materials Data for Cyclic Loading. Elsevier, Amsterdam, The Netherlands.

Bonewald LF (2011) The amazing osteocyte. J Bone Miner Res 26: 229-238.

Burr DB (2002) Targeted and nontargeted remodeling. Bone 30: 2-4.

Burr DB, Martin RB (1993) Calculating the probability that microcracks initiate resorption spaces. J Biomech 26: 613-616.

Colopy SA, Benz-Dean J, Barrett JG, Sample SJ, Lu Y, Danova NA, Kalscheur VL, Vanderby Jr R, Markel 
MD, Muir P (2004) Response of the osteocyte syncytium adjacent to and distant from linear microcracks during adaptation to cyclic fatigue loading. Bone 35: 881-891.

Cowin SC (2002) Mechanosensation and fluid transport in living bone. J Musculoskel Neuron Interation, 2: 256260.

Cowin SC, Moss-Salentijn L, Moss ML (1991) Candidates for the mechanosensory system in bone. $\mathrm{J}$ Biomech Engi 113: 191-197.

Dooley C, Tisbo P, Lee T, Taylor D (2012) Rupture of osteocyte processes across microcracks: the effect of crack length and stress. Biomech Model Mechanobiol 11: 759-766.

Dunstan CR, Somers NM, Evans RA (1993) Osteocyte death and hip fracture. Calcii Tissue Int 53: S113-S117.

Eriksen EF, Langdahl B (1995) Bone changes in metabolic bone disease. Acta Orthopaed Scand 266: 195 201.

Frost HM (1960) Presence of microscopic cracks invivo in bone. Bull Henry Ford Hosp 8: 25-35.

Frost HM (1987) Bone "mass" and the "mechanostat": A proposal. Anat Rec 219: 1-9.

Hazenberg JG, Freeley M, Foran E, Lee TC, Taylor D (2006) Microdamage: A cell transducing mechanism based on ruptured osteocyte processes. J Biomech 39: 2096-2103.

Jilka RL (2003) Biology of the basic multicellular unit and the pathophysiology of osteoporosis. Med Pediat Oncol 41: 182-185.

Kennedy OD, Herman BC, Laudier DM, Majeska RJ, Sun HB, Schaffler MB (2012) Activation of resorption in fatigue-loaded bone involves both apoptosis and active pro-osteoclastogenic signaling by distinct osteocyte populations. Bone 50: 1115-1122.

Kuznetsova TG, Starodubtseva MN, Yegorenkov NI, Chizhik SA, Zhdanov RI (2007) Atomic force microscopy probing of cell elasticity. Micron 38: 824-833.

Lee TC, Staines A, Taylor D (2002) Bone adaptation to load: microdamage as a stimulus for bone remodelling. J Anat 201: 437-446.

Malone AMD, Anderson CT, Tummala P, Kwon RY, Johnston TR, Stearns T, Jacobs CR (2007) Primary cilia mediate mechanosensing in bone cells by a calciumindependent mechanism. Proc Natl Acad Sci USA 104: 13325-13330.

Mofrad MHK, Kamm RD (2010) Cellular Mechanotransduction. Cambridge University Press, Cambridge, UK.

Mori S, Harruff R, Ambrosius W, Burr DB (1997) Trabecular bone volume and microdamage accumulation in the femoral heads of women with and without femoral neck fractures. Bone 21: 521-526.

Mulcahy LE, Taylor D, Lee TC, Duffy GP (2011) RANKL and OPG activity is regulated by injury size in networks of osteocyte-like cells. Bone 48: 182-188.

Mullender MG, Huiskes R, Versleyen H, Buma P (1996) Osteocyte density and histomorphometric parameters in cancellous bone of the proximal femur in five mammalian species. J Orthopaed Res 14: 972-979.

Nichol JA, Hutter OF (1996) Tensile strength and dilatational elasticity of giant sarcolemmal vesicles shed from rabbit muscle. J Physiol 493: 187-198.
Noble BS (2003) Mechanical loading: Biphasic osteocyte survival and targetting of osteoclasts for bone distruction in rat cortical bone. Am J Physiol Cell Physiol 284: 934-943.

Noble BS, Stevens H, Loveridge N, Reeve J (1997) Identification of apoptotic changes in osteocytes in normal and pathological human bone. Bone 20: 273-282.

O'Brien FJ, Taylor D, Dickson GR, Lee TC (2000) Visualisation of three-dimensional microcracks in compact bone. J Anat 197: 413-420.

O'Brien FJ, Taylor D, Lee TC (2002) An improved labelling technique for monitoring microcrack growth in compact bone. J Biomech 35: 523-526.

Schaffler MB, Jepsen KJ (2000) Fatigue and repair in bone. Int J Fatigue 22: 839- 846.

Schaffler MB, Pitchford WC, Choi K, Riddle JM (1994) Examination of compact bone microdamage using backscattered electron microscopy. Bone 15: 483-488.

Sheetz MP, Sable JE, Dobereiner HG (2006 ) Continuous membrane-cytoskeleton adhesion requires continuous accommodation to lipid and cytoskeleton dynamics. Ann Rev Biophys Biomol Struct 35: 417-434.

Taylor D (2011) What we can't learn from nature. Mater Sci Eng 31: 1160-1163.

Taylor D, Hazenberg JG, Lee TC (2003) The cellular transducer in damage-stimulated bone remodelling: a theoretical investigation using fracture mechanics. J Theor Biol 225: 65-75.

Taylor D, O'Brien F, Prina-Mello A, Ryan C, O'Reilly P, Lee TC (1999) Compression data on bovine bone confirms that a "stressed volume" principle explains the variability of fatigue strength results. Journal of Biomechanics 32:1199-1203.

Temiyasathit S, Jacobs CR (2010) Osteocyte primary cilium and its role in bone mechanotransduction. Ann NY Acad Sci 1192: 422-428.

Verborgt O, Gibson GJ, Schaffler MB (2000) Loss of osteocyte integrity in association with microdamage and bone remodeling after fatigue in vivo. J Bone Miner Res 15: 60-67.

You J, Yellowley CE, Donahue HJ, Zhang Y, Chen Q, Jacobs CR (2000) Substrate deformation levels associated with routine physical activity are less stimulatory to bone cells relative to loading-induced oscillatory fluid flow. J Biomech Eng 122: 387-393.

You J, Reilly GC, Zhen X, Yellowley CE, Chen Q, Donahue HJ, Jacobs CR (2001) Osteopontin gene regulation by oscillatory fluid flow via intracellular calcium mobilization and activation of mitogen-activated protein kinase in MC3T3-E1 osteoblasts. J Biol Chem 276: 1336513371.

\section{Discussion with Reviewers}

D. Burr: It is not clear how the authors know that what they are calling processes are in fact that, and not some other component of the matrix (e.g., osteopontin, "bone glue", etc.). They look very much like what Hansma (and Ritchie) call "bone glue" which is found in microcracks. 
Although others have identified processes conclusively by phalloidin staining, that is not done here. Please comment! Authors: Previous work by our group used phalloidin staining to demonstrate that these features are cellular processes (Hazenberg et al., 2006, text reference) It is true that other features can sometimes be seen in microcracks, such as collagen fibres, "bone glue" etc. However cellular processes are morphologically different from these other features so we are confident that we can recognise them when viewing them under the SEM. A second researcher verified the initial counts to rule out individual bias.

M. Schaffler: The bone "microdamage" studied in these investigations is a crack (microcrack or macrocrack?) propagating from a notched toughness type specimen loaded cyclically. It is difficult to appreciate the physiological relevance of such induced damage foci compared to true fatigue microcracks within bone. They differ in size, length, number and mechanics of their production. Please comment!

Authors: It is true that the microcracks studied in the in situ strained bone were somewhat different from microcracks in their native state in vivo. However, all the other tests described in this paper were conducted on naturally occurring microcracks. We used microcracks which had formed in vivo and then, in some tests, loaded these cracks to cause further process rupture. Thanks to our staining technique we knew that we were always looking at naturally-occurring cracks. The experiment conducted in situ in the SEM used a different method for creating the crack, resulting in cracks which were slightly longer than typical microcracks but had similar crack openings and similar numbers of broken and unbroken processes. So we feel confident in stating that the relationship between crack opening and number of cycles to failure will also apply to natural cracks. 